\title{
Prolonged motor skill learning - a combined behavioural training and theta burst TMS study
}

\author{
Thomas Platz ${ }^{\mathrm{a}, *}$, Sybille Roschka ${ }^{\mathrm{a}}$, Karla Doppl ${ }^{\mathrm{a}}$, Charlotte Roth ${ }^{\mathrm{a}}$, Martin Lotze $^{\mathrm{b}}$, \\ Alexander T. Sack ${ }^{\mathrm{c}}$ and John C. Rothwell ${ }^{\mathrm{d}}$ \\ ${ }^{a}$ BDH-Klinik Greifswald, Neurorehabilitation Centre and Spinal Cord Injury Unit, \\ Neuroscience Department, Ernst-Moritz-Arndt-Universität, Greifswald, Germany \\ ${ }^{\mathrm{b}}$ Functional Imaging Department, Center for Diagnostic Radiology and Neuroradiology, \\ Ernst-Moritz-Arndt-Universität, Greifswald, Germany \\ ${ }^{\mathrm{c}}$ Department of Cognitive Neuroscience, Faculty of Psychology and Neuroscience, \\ Maastricht University, The Netherlands \\ ${ }^{\mathrm{d}}$ Sobell Department of Motor Neuroscience and Movement Disorders, Institute of Neurology, \\ Queen Square, London, UK
}

\begin{abstract}
Purpose: To assess the behavioural effects of prolonged motor practice in healthy volunteers, and the specific impact of inhibiting different motor-related brain regions in the late phase of motor learning using continuous theta burst transcranial magnetic stimulation (cTBS).

Methods: Twelve subjects trained their non-dominant arm in eight arm motor tasks (Arm Ability Training, AAT) once a day for three weeks (16 sessions). During the last four days, training was performed before and after applying cTBS to either M1, S1, SMA, or PMC.

Results: The AAT induced substantial and robust motor learning for the trained arm with variations across tasks. Considerable motor learning was also observed in the non-trained dominant arm with remarkably similar variations across tasks, suggesting that practise improved common underlying sensorimotor capacities (abilities) in addition to effector-specific effects. When applied after prolonged training, inhibitory cTBS showed no detrimental effects on motor performance/learning; M1 cTBS even improved performance in a labyrinth task.

Conclusions: Prolonged training with the non-dominant arm led to profound motor learning across abilities with transfer to the non-trained dominant arm. Unlike during early stages of motor learning, no detrimental effect of cTBS over M1, S1, PMC, or SMA could be substantiated after prolonged motor practice.
\end{abstract}

Keywords: Motor practice, learning, cortex, plasticity, transcranial magnetic stimulation

${ }^{*}$ Corresponding author: Prof. Dr. med. Thomas Platz, BDHKlinik Greifswald, An-Institut der Ernst-Moritz-Arndt-Universität, Karl-Liebknecht-Ring 26a, D-17491 Greifswald, Germany. E-mail: t.platz@bdh-klinik-greifswald.de.

\section{Introduction}

With sufficient practice we learn to perform skills under a variety of conditions, even if they have never been encountered before. Schema theory suggests that this implies acquisition of a set of underlying 
relationships between components of the task which we can define as a "generalised motor program" (GMP) that stores the invariant features that control movement production (Maas et al., 2008; Keetch et al., 2008). It is assumed that a skilled performer retrieves a GMP for a class of learnt skills and then adjusts parameters to suit the specific environmental demands.

Compatible with the notion of the specificity of learning principle are observations from functional imaging studies indicating that motor skill learning is a dynamic process that involves plastic changes in a range of cortical and subcortical areas, with task-specific variations. Cortical areas including the primary motor cortex (M1), primary somatosensory cortex (S1), premotor cortex (PMC), the supplementary motor area (SMA), the posterior parietal cortex (PPC) and the cerebellum seem to be differentially involved in performance and learning of different motor tasks with different demands for sensorimotor integration (Ashe et al., 2006; Grafton et al., 2002; Park et al., 2010). The primary motor cortex (M1) could be essential for the storage of newly learned motor skills (Lu and Ashe 2005; Matsuzaka et al., 2007), a similar role has also been attributed to those parts in the basal ganglia that belong to the sensorimotor cortico-basal ganglia circuit (Jueptner et al., 1997; Lehéricy et al., 2005; Toni et al., 2001). A network involving the dorsal premotor cortex (PMd) and the posterior parietal cortex (PPC) seems to be relevant for planning and selection of learned visuomotor associations (Grafton et al., 1998; 2002; Grol et al., 2006). The temporal representation of learned associations as in sequential movements might critically involve the supplementary and presupplementary motor areas (SMA and pre-SMA), with the SMA showing selectivity for the type of movements performed and the pre-SMA for grouping and organization such as rank order of sequential movement elements (Isoda and Tanji 2004; Shima and Tanji 2000).

The specificity with which brain network are activated in motor learning experiments varies according to the stage of motor learning: different patterns are seen during the early stage of learning when a skill is first acquired and the late stage after long-term practice (Seidler, 2010). Using positron emission tomography (PET), Seitz and Roland (1992) revealed that subjects learning a complex finger sequence task show an increased activity of motor cortical areas only during early stages of learning, but not when the sequence had been learnt. After prolonged practise, the basal ganglia became more activated when the learnt sequence was performed. In a similar vein, more recently it has been shown, that the entire associative anterior premotor cortico-basal ganglia circuit is activated during the early stages of learning, but that once learned after extended practice, motor skills would then be stored in the sensorimotor cortico-basal ganglia circuit (Lehéricy et al., 2005).

The present experiment was designed to assess the behavioural effects of prolonged motor practice, and the specific impact of inhibiting different motor-related brain regions in the late phase of motor learning when healthy subjects performed a multi-task motor training, the Arm Ability Training (AAT) that had previously been developed for brain-damaged patients with proven clinical effectiveness (Platz et al., 2001; Platz et al., 2009).

The eight different arm motor tasks of the AAT are thought to address different independent sensorimotor abilities, e.g. aiming, steadiness, speed, dexterity (Platz, 2004; Platz et al., 2012), that are practiced over a course of three weeks in the clinical setting. The training implements a variety of aspects that facilitate motor learning, i.e. maintenance (retention) and generalization (transfer) of practice effects (Schmidt and Lee, 2005): large amount of repeated daily practice over three weeks, blocked practice for classes of movements with variation of task difficulty within those classes, external focus of attention with emphasis on movement results supported by intermittent augmented feedback in the form of knowledge of result.

In a previous related experiment focusing on early stages of motor learning, healthy subjects, practiced the AAT tasks with their non-dominant left hand for only 5 consecutive days (Platz et al., 2012). In that experiment, substantial motor learning was observed and yet the degree of motor skill improvement varied across tasks favouring the notion of different learning dynamics across tasks. In accordance with the assumption that early motor learning involves cortical sensory and motor areas, inhibitory rTMS (cTBS, continuous theta burst stimulation) over S1, M1, and PMC significantly impaired within session learning dynamics and did so with effects that varied across tasks, indicating that these cortical areas functionally contribute differentially to early motor learning. Concretely, cTBS over S1 was detrimental for all practiced tasks whereas cTBS over M1 specifically impaired rapid 
tapping movements, and cTBS over PMC training of ballistic arm navigation in extra-personal space.

The current experiment addressed the question of behavioural effects of more prolonged motor practice in another sample of healthy subjects, again practicing the eight different arm motor tasks of the AAT with their non-dominant arm, but over a period of three weeks. Several questions were of interest: would prolonged practice lead to more pronounced training effects as compared to our previous findings on early stages of motor learning? Could differential dynamics across tasks again be documented? Would training induce transfer of improved skilfulness to a non-trained motor task? Would the effects of practice transfer to the non-trained dominant arm? If that was the case, this would imply that motor practice did not only induce effector-specific motor learning, but improvement of motor abilities that were (at least partially) effector-independent. With regard to the functional role of cortical sensory and motor areas for late motor learning, we here also assessed whether using the same cTBS as in the previous experiment on early motor learning dynamics (Platz et al., 2012), but now only applied after prolonged motor practice in the last week of training would again induce similar detrimental effects on training dynamics. Based on the above reported findings (Seitz and Roland, 1992; Lehéricy et al., 2005) and the thus assumed reduced relevance of cortical areas in the late stages of motor learning, we expected that the previously shown detrimental effects of cTBS over motor-related cortical areas would vanish when applying cTBS after extended practise, despite using the same training tasks, the same cTBS protocol, and target sites (M1, S1, SMA, or PMC) in cTBS-naïve subjects.

\section{Materials and methods}

\subsection{Subjects}

Twelve right-handed AAT-naïve volunteers of (5 women, 7 men; mean age $24.4 \pm 3.2$ years; Oldfield Handedness laterality ratio: $95 \pm 6$ ) (Oldfield, 1971) participated and completed the study. All participants had normal or corrected-to-normal vision and had no documented history of neurological or psychiatric disorders when they were enrolled. They gave their written informed consent after being introduced to the study objectives and procedures. The study was approved by the local Medical Ethical Commission of Ernst-Moritz-Arndt University, Greifswald, where the experiment was conducted.

\subsection{Overall study design}

Subjects performed a specific daily arm training (AAT) for 16 sessions over a 3-week-period. At baseline, and after each week of training, all training tasks were tested with both the trained non-dominant left arm and the non-trained dominant right arm.

During the final week, training was performed before and after applying an inhibitory form of repetitive transcranial magnetic stimulation (cTBS, continuous theta burst) to either M1, S1, SMA, or PMC.

\subsection{Motor tasks and training}

The AAT consists of eight different motor tasks that are thought to train different abilities such as speed, aiming, dexterity, tracking and steadiness: the training tasks with a fixed sequence within each training session are aiming, tapping, crossing circles, turning coins, labyrinth tracking, bolts and nuts, placing small objects, and placing large objects (compare Fig. 1 in Platz et al., 2012).

Work load had been standardised for all participants in advance: every day the same amount of task repetitions had to be trained. The specified number of repetitions for each task per day had been divided in four equal "blocks" (fixed number of repetitions lasting approximately one minute at the beginning). During training, participants were continuously encouraged to try to fulfil their workload in even shorter time, but without compromise of the individual tasks' accuracy demands. Any progress was shown to participants for each type of task during training sessions using diagrams on a PC screen (Arm Ability software; knowledge of result, i.e. time needed for blocks of task repetitions). Participants repeated a total of four blocks of repetitions for each training task per day in two consecutive training slots with two combined blocks for each task for all tasks (1st half of training session) followed by another two combined blocks for each task for all tasks (2nd half of training session) (compare Fig. 1A). The same standardised training was applied for 16 weekdays, each training lasting approximately one hour, over three weeks (compare Fig. 1B). 


\section{A. Structure of blocked training each day}

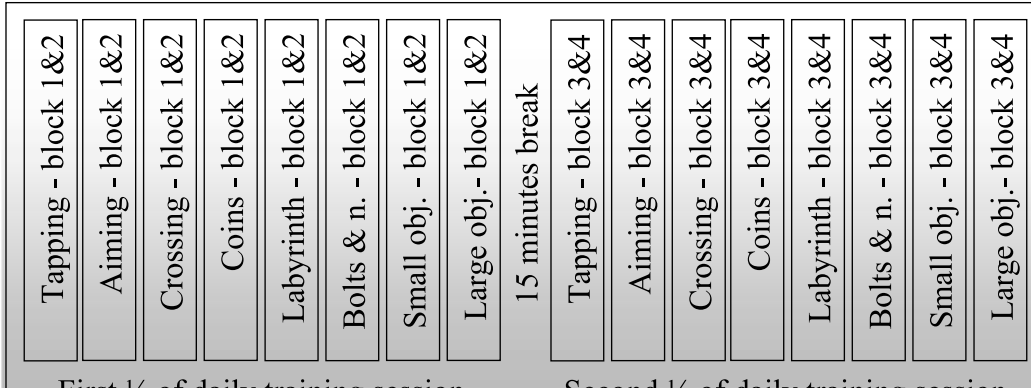

First $1 / 2$ of daily training session

Second $1 / 2$ of daily training session

\section{B. Sequence of daily training and weekly tests}

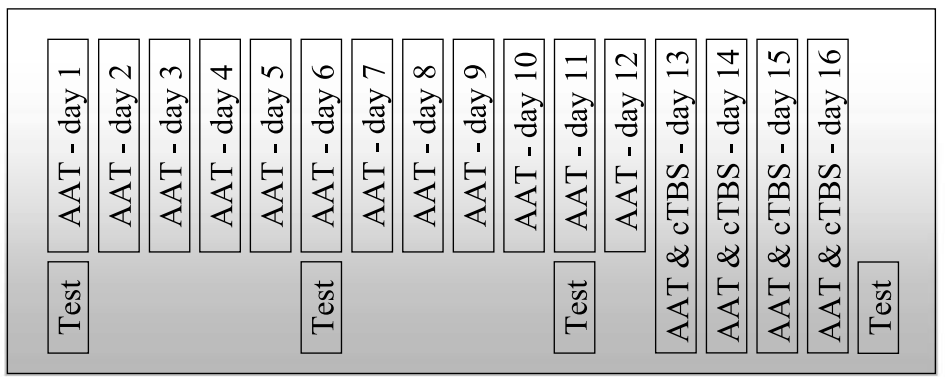

\section{Last week of training - cTBS}

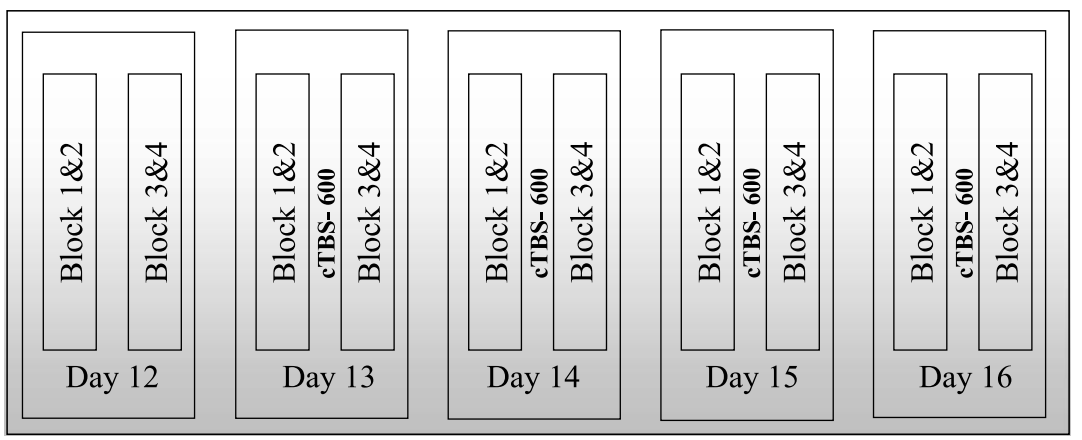

Fig. 1. Sequence of experimental events. (A) Structure of Arm Ability Training (AAT) sessions: Participants repeated a total of four blocks of repetitions for each training task per day in two consecutive training slots with two combined blocks for each task for all tasks (1st half of training session) followed by another two combined blocks for each task for all tasks (2nd half of training session). (B) The same standardised training was applied for 16 weekdays over three weeks. Each training session lasted approximately one hour. (C) During the last four sessions on days 13-16 of the training, the participants performed the AAT before and after having received cTBS to one of the four motor-related target sites using MRI-guided TMS neuronavigation.

\subsection{Neuronavigation and TMS procedure}

\subsubsection{Brain cortex modeling and determination of stimulation sites}

We obtained anatomical brain measurements of all participants using magnetic resonance imaging (MRI). An anatomical T1-weighted three-dimensional Mag- netization Prepared Rapid Gradient Echo (MPRAGE) image was acquired for each subject at a 3T Siemens Magnetom Verio (Siemens, Erlangen, Germany) equipped with a 32-channel head coil, voxel size was $1 \times 1 \times 1 \mathrm{~mm}^{3}$. We performed a surface reconstruction to recover the spatial structure of the cortical sheet based on the white-grey-matter boundary using 
Brain Voyager $^{\mathrm{TM}}$ TMS neuronavigation software (TMS Neuronavigator edition of BrainVoyager QX 2.1 by Brain Innovation B.V., Maastricht, NL). We then identified four motor-related brain regions, namely M1, S1, SMA, and PMC, on the basis of each individual brain gyrification: Motor evoked potentials (MEPs) were used to determine the coil position over M1 ("hot spot") that evoked the best response in the left (and right) abductor pollicis brevis muscle (APB). The other stimulation sites of the right brain were defined in the individual MR data: PMC, in the middle of the gyrus immediately frontal to $\mathrm{M} 1$ on a line perpendicular to the central gyrus through the hot spot; S1, in the middle of the gyrus immediately posterior to M1 on a line perpendicular to the central gyrus through the hot spot; SMA, $3 \mathrm{~cm}$ anterior from the leg motor area (mid of precentral gyrus $1 \mathrm{~cm}$ lateral to midsagittal line) and $1 \mathrm{~cm}$ lateral to the midsagittal line. The locations of M1, S1, PMC and SMA were maintained both within and across sessions by use of the BrainVoyager ${ }^{\mathrm{TM}}$ TMS neuronavigation (compare Fig. 3 in Platz et al., 2012).

\subsection{2. $T M S$}

Each participant's anatomical MRI and head and brain surface models were used for stereotaxic coregistration of the participant's brain with the TMS coil. This enabled online control and re-test-reliability of coil positioning during each session and across days. Subjects were seated in a reclining chair and instructed to remain relaxed throughout the application of rTMS. Surface electromyography (EMG) from participants' APB was monitored using the motor evoked potential unit of (Dantec Keypoint ${ }^{\circledR}$ by Alpine Biomed ApS, Skovlunde, DK).

Application of TMS was performed with a $75 \mathrm{~mm}$ figure-8 passively cooled coil (MCF-B65) and the MagPro X100 Magnetic Stimulator and (MagVenture A/S, Farum, DK). The TMS coil was oriented tangentially to the scalp with the handle pointing back and away from midline at $45^{\circ}$ during stimulation of both M1 (for thresholding and cTBS), S1 (for cTBS), and PMC (for cTBS) while it was oriented tangentially to the scalp with the handle pointing downward and away from midline at $90^{\circ}$ during stimulation of the SMA (for cTBS).

Each rTMS session consisted of a continuous $40 \mathrm{sec}-$ ond train of TBS with 600 stimuli (cTBS-600). cTBS protocols apply short bursts (3 stimuli) of $50 \mathrm{~Hz}$ rTMS which are repeated at a rate in the theta range $(5 \mathrm{~Hz})$. In this experiment, an intensity that equals $80 \%$ of the active motor threshold was applied; the AMT being the intensity that evokes an MEP of $\geq 200 \mu \mathrm{V}$ in $\geq 5$ out of ten trials while the subjects perform an isometric contraction at a level of $20 \%$ of her/his maximum voluntary contraction. Applied continuously (cTBS), the net effect of TBS is inhibitory (Huang et al., 2005), cTBS600 can temporarily suppress local cortical excitability for about 60 minutes. Since this protocol had shown detrimental effects on early motor learning in a parallel previous study (Platz et al., 2012), it was decided to apply cTBS only during the primary period of interest, i.e. after extended practice, in this study. Thereby, cTBS-naive subjects could be investigated at this later stage of motor learning with the same procedure. In these four sessions (on days 13-16 of the training), the participants performed the AAT before and after having received cTBS to one of the four above mentioned motor-related target sites using MRI-guided TMS neuronavigation (compare Fig. 1C). The order of stimulation site was counterbalanced across subjects.

\subsection{Data analysis}

\subsubsection{Motor behaviour data}

The behavioural outcome measures for each single training session were the time needed for each block (blocks 1 to 4 ) for each training task of the AAT (tasks 1 to 8) for each day of practice. These outcome measures (in seconds; approximately 60 seconds per block) had been standardised with baseline data from day 1 . Thus, baseline data for each task were 1.0; any improvement in time needed to perform the training tasks would reduce the standardised block measure below 1.0. For the analysis of motor learning effects, these standardised scores tasks were used since they were comparable across tasks.

\subsubsection{Weekly motor behaviour analysis}

The weekly motor behaviour analysis was based on averaged data for a given motor tasks of the AAT tasks 1 to 8 per day (average of 4 blocks for each task). On day 1 of training and on days 6,11 , and 16 , i.e. after 1,2 and 3 weeks of training the performance with the AAT tasks was assessed for both the trained left and non-trained right arm. In addition, the Nine-Hole-PegTest (Mathiowetz et al., 1985) was administered as a standard motor test for both hands on these days. 


\subsubsection{Motor behaviour before or after cTBS}

Participants had been informed about the different stimulation sites used; with regard to any expected effects of cTBS they had been told that effects - if any - would be quite small and only detectable in group statistics. Since cTBS was applied on days 13 to 16 between the first and second half of the training session (i.e. between blocks $1 \& 2$ and $3 \& 4$ for each task), effects of cTBS were analysed based on these differences: for each day and task, difference scores between blocks $1 \& 2$ and $3 \& 4$ were calculated and standardised (with baseline data). Thus, for testing the effects of stimulation per site, pre and post stimulation data (blocks $1 \& 2=$ pre stim, blocks $3 \& 4=$ post stim) and their differences were calculated for each site of stimulation and each training task. The sequence of site of stimulation (i.e., M1, S1, PMC, SMA) across days had been counterbalanced across subjects. In addition, the Nine-Hole-Peg-Test was administered as a standard motor test for both hands after the AAT tasks, both before and after cTBS. The time interval between the first and second half of the training was 15 minutes.

\subsubsection{Statistical analyses}

General linear models within a repeated-measures ANOVA design were used to assess both the effect of (a.) training and (b.) cTBS on outcome measures: repeated measures were either (a.) standardised time scores for the weekly assessment of motor tasks or (b.) pre vs. post stim differences for the cTBS sites.

For the analysis of training effects, a repeatedmeasures ANOVA design was used to assess the effect of training across weeks ( 0 to 3 ), across tasks (task 1 to 8), and hand (left trained versus right non-trained) on the timed performance. Embedded in this overall ANOVA model was a Helmert transformation that compared each level of week ( 0 to 3 ) to the mean of subsequent levels indicating the point when performance ceased to change from week to week.

Effects of cTBS site were analysed with a repeatedmeasures ANOVA design that used change scores from block $1 \& 2$ to block $3 \& 4$. Embedded in this overall ANOVA model were specific contrasts between each stimulation site and the mean of all other stimulation sites (transformation matrix) and thus specific differences for a given stimulation site were determined: all other stimulation sites served as control condition for each stimulation site.

$F$ values presented for these models are partial $F$ values (based on type III sums of squares). Effect sizes "d" had been calculated for statistically significant main effects of interest based on these F statistics; by convention, they had been considered small $\mathrm{d}=0.2$, medium $d=0.5$, or large $d=0.8$ (Cohen, 1988). In case assumptions of the repeated measures ANOVA were violated (according to sphericity testing), Huynh-Feldt epsilon adjusted $p$ values (labeled "H-F") were used. Alpha was set at 0.05 .

\section{Results}

\subsection{Motor learning}

\subsubsection{Arm Ability tasks}

The analysis of changes in task performance (based on all tasks, weeks $0-3$, and both arms) indicated that considerable motor learning occurred across weeks $(\mathrm{F}(3,33)=282.17 ; p<0.0001 ; \mathrm{d}=5.06)$ and for both arms, while being bigger for the trained left arm ('side $\times$ week' interaction: $(F(3,33)=24.74$; $p<0.0001$ ) (compare Fig. 2). For the trained left arm, the improvement from baseline was on average $33,8 \%$ indicating substantial and robust motor learning over the course of three weeks. While becoming smaller over time, the Helmert transformation nevertheless indicated that the level of performance improved significantly from week to week throughout the 3 week course of training (week 1: $\mathrm{F}(1,11)=1680.78 ; p<0.0001 ; \mathrm{d}=12.36$; week 2 : $\mathrm{F}(1,11)=29.31 ; p=0.0002 ; \mathrm{d}=1.63$; week 3 : $\mathrm{F}(1,11)=9.33 ; p=0.011 ; \mathrm{d}=0.92)$.

The dynamics of motor skill learning was different across tasks as indicated by a significant 'task $\times$ week' interaction $(\mathrm{F}(21,231)=2.64 ; p=0.0002)$ and a main effect of 'task' $(\mathrm{F}(7,77)=3.79 ; p=0.0014)$ (even though all data had been standardised with performance of day 1 for each task), suggesting a varying degree of skill level acquisition over the course of training. Table 1 shows the different levels of performance at the end of training. The tapping, turning coins, and placing small objects tasks showed the least improvement (23-28\% on average), the nuts and bolts, labyrinth, and aiming tasks the largest dynamics in motor skill learning (37-40\% improvement on average). The pattern of dynamics was, however, comparable for both the trained left and non-trained right arm ('side $\times$ task $\times$ week' interaction: $(\mathrm{F}(21,231)=1.21$; n.s. $)$.

When only data for the non-trained right arm was analysed, considerable motor learning across 

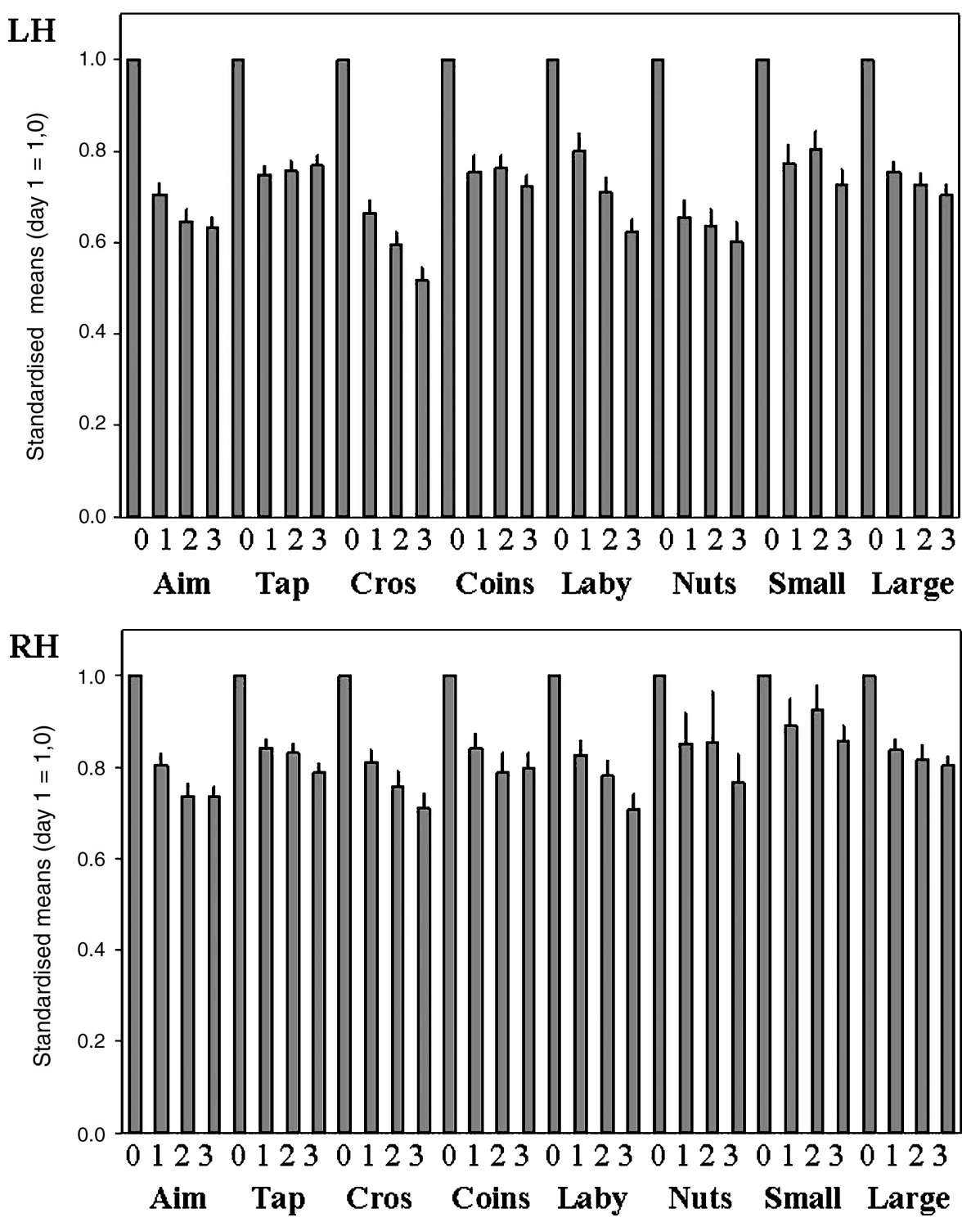

Fig. 2. Arm Ability Training tasks. The Arm Ability Training consists of 8 different motor tasks that are thought to train different abilities such as speed, aiming, tracking, steadiness and dexterity. The tasks are aiming (Aim), tapping (Tap), crossing circles (Cros), turning coins (Coins), labyrinth tracking (Laby), bolts and nuts (Nuts), placing small objects (Small), and placing large objects (Large). Shown is average data for all eight Arm Ability tasks presented as mean and standard error of the mean (error bars) for 4 assessments per tasks, i.e. at baseline ( 0 ), after one (1), two (2), and three (3) weeks of training, respectively. Data has been standardised with baseline data (baseline $=1$; values below 1.0 denote improved performance) and is separately presented for the trained non-dominate left hand (LH) and the non-trained dominant right hand (RH). The statistical analysis of these changes corroborated that motor learning occurred for both hands, but to a varying degree across tasks (for details see text).

weeks could also be corroborated $(\mathrm{F}(3,33)=95.02$; $p<0.0001 ; \mathrm{d}=2.94)$. While again being different across tasks, the pattern of motor learning was remarkably similar between the trained left and the non-trained right arm (compare Fig. 2).

\subsubsection{Performance with the non-trained Nine-Hole-Peg-Test (NHPT)}

The ANOVA for repeated measures indicated an improved performance of this non-trained task across weeks for both the trained left and non-trained right 
Table 1

Final performance with the Arm Ability Training tasks after 3 weeks of training

\begin{tabular}{lc}
\hline Task & Mean 95\% CI \\
\hline Aiming & $0.63(0.58,0.69)$ \\
Tapping & $0.77(0.72,0.81)$ \\
Crossing circles & $0.52(0.46,0.57)$ \\
Turning coins & $0.72(0.66,0.78)$ \\
Labyrinth & $0.62(0.56,0.68)$ \\
Nuts and bolts & $0.60(0.51,0.69)$ \\
Placing small obj. & $0.73(0.66,0.80)$ \\
Placing large obj. & $0.71(0.66,0.75)$ \\
\hline
\end{tabular}

Table 1 depicts the performance for each of the 8 Arm Ability Training tasks at the end of training (end of week 3 ). Presented are mean values of standardised scores and the $95 \%$ confidence intervals. Standardisation was done with scores from each task at the beginning of training (day 1). Values $<1.0$ indicate improved performance.

arm (mean and 95\% CI of time needed for the NHPT: baseline right hand 16,7 s $(15.3,18.0)$, left hand 17,4 s (16.2, 18.6); week 3 right hand 14,3 s $(13.3,15.3)$, left hand $15,1 \mathrm{~s}(14.1,16.1)$ (factor 'week', $\mathrm{F}(3,33)=8.83$; $p=0.0006 ; \mathrm{d}=0.90)$. Neither overall performance nor improvement over 3 weeks on this task was statistically significantly different for the right or left hand (factor 'side' and interaction 'side $\times$ week': n.s.).

\subsection{Effects of cTBS}

Effects of cTBS when applied during the last week of training were analysed as differences that occurred within each session from the 1 st half of the session (blocks $1 \& 2$ for each task) to the 2 nd half of the session (blocks $3 \& 4$ for each task) after cTBS, with negative values indicating improvement rates and positive values indicating deterioration.

On the day before the first cTBS (on day 12 of the training), these intra-session changes had on average already become relatively small with a grand average change rate of -0.017 (95\% CI: 0.002--0.035), indicating a within-session improvement of $1.7 \%$ on average from the 1 st to the 2 nd half of each training session.

When the grand average of change scores across all 8 training tasks was analysed, the repeated measures ANOVA did not substantiate any differential influence of cTBS over different cortical areas on the mean improvement rates within each session $(\mathrm{F}(4,44)=0.42$; $p=0.7950$ ) (compare Fig. 3).

The effect of cTBS over different stimulation sites on change scores was also analysed for each of the AAT tasks separately.

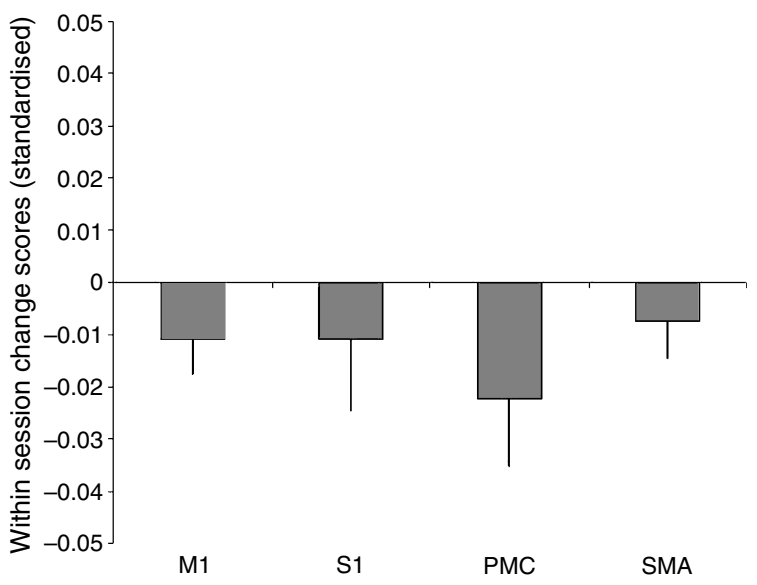

Fig. 3. Effects of cTBS. Figure 3 presents grand average data across all eight Arm Ability tasks. Data is presented as mean and standard error of the mean (error bars) of changes scores from the 1st half of the training session before cTBS to the 2 nd half of a training session after cTBS. Negative values indicate an (continued) improvement despite the cTBS, positive values would have indicated deterioration of performance after cTBS. Differential effects of cTBS over different brain sites (i.e. M1, S1, PMC, and SMA) had been analysed statistically; the data did not indicated differential effects of cTBS over M1, S1, PMC, or SMA as compared to the other stimulation sites.

Aiming, tapping, crossing circles, turning coins, bolts and nuts, and placing small or large objects were all not statistically differentially affected by the site of stimulation.

A differential effect was observed for the labyrinth task: the contrast for cTBS over M1 (as compared to all other stimulation sites) showed a beneficial influence on performance of the labyrinth task $(\mathrm{F}(1,11)=5.40$; $p=0.0403$ ). Since the cTBS data had been used a second time for this analysis, a Bonferroni correction for the risk of type I errors would imply a level of alpha of 0.025 questioning the statistical significance of this result.

In addition, there was no evidence that the Nine-Hole-Peg-Test performed with either hand was differentially affected by the different cTBS sites.

\subsection{Side effects}

One subject that volunteered to participate had mild hypotensive symptoms during single pulse TMS and was consecutively excluded from the study (before cTBS application).

One female subject with an (initially not reported) history of migraine had mild headaches before the first 
TMS/cTBS session that were not changed immediately thereafter (morning), developing mild migraine headaches in the afternoon (day 1 of TMS/cTBS only) treated with rest and $500 \mathrm{mg}$ paracetamol orally by herself. Another male subject noted mild headaches with an occipital-frontal bilateral distribution on the evening of the first stimulation day only with some concurrent subjective change of concentration on that day. Three other subjects reported mild headaches on stimulation day 2 (2 male subjects, in the evening) or stimulation day 3 (1 female subject, starting at noon). All these subjects did not see a need to treat their mild headaches during that episode. All subjects experiencing an episode of headaches wanted to continue their participation in the project and had no more episodes of headaches.

\section{Discussion}

\subsection{Motor learning}

From a clinical point of view it is of major importance to know whether training schedules induce robust motor learning, i.e. "relatively permanent changes in the capability for movement" (Schmidt and Lee, 2005, p. 302). The AAT design incorporates a variety of principles that may support motor learning both in terms of maintenance (retention) and generalisation (transfer) of training effects.

The behavioural data shows that AAT not only improves performance of patients with reduced fine motor skill after stroke (Platz et al., 2009), but also improves considerably performance of young healthy subjects who train their non-dominant arm over a prolonged period of 3 weeks (effect size 5.06). At the end of training, standardised scores had on average improved between 23 and $40 \%$ for the different motor tasks indicating a considerable and robust improvement in performance. Tasks that afforded speed of finger movements (tapping) or dexterity (turning coins, placing small objects) showed lower overall improvement rates and more floor effects than tasks requiring steadiness (crossing circles) and visuomotor integration (aiming, labyrinth task). The latter might therefore be more susceptible to effects of prolonged training.

Thus, a stable and considerable improvement of performance had been observed over several weeks when performance was assessed outside the training sessions (compare Fig. 2). Accordingly, retention of practice effects for the practiced tasks and thus one aspect of motor learning could be inferred for a number of classes of movements that had been trained in parallel.

Since improved performance in trained tasks does not in itself imply generalisation of training effects, it was further of interest to know whether prolonged practice of the AAT induced transfer of training effects to a non-trained task and/or the non-trained limb and thus generalisation effects of motor learning.

The behavioural evidence indeed suggested a transfer of improved skilfulness, for one to a non-practiced task (NHPT) (effect size 0.90), and second to performance of the non-trained right arm (effect size 2.94) and thus convincingly supported the notion of AAT-induced motor learning in terms of generalisation (transfer).

Transfer of learning with one arm to performance of the same task with the other arm has previously been reported for both healthy subjects (Grafton et al., 2002; Stockel and Weigelt, 2011) and Parkinson patients (Platz et al., 1998). A novel finding here was that this transfer of improved skilfulness had been achieved when a variety of motor tasks that had previously been shown to address independent sensorimotor abilities (Platz et al., 2012) had been practiced in parallel. The observed transfer of skilfulness to the non-trained limb and the remarkable similarity of the pattern of these changes for either hand (compare Fig. 2) are suggestive of common resources that had been used for each class of movements with either hand and were improved by practice. The findings would be compatible with the theoretical notion of "generalised motor programs" (GMP) that store the invariant features that control movement production for each class of motor skills trained (Schmidt, 1975; Schmidt and Lee, 2005; Keetch et al., 2008; Maas et al., 2008).

Performance with the right arm certainly requires a transformation for the different effectors used accounting for the incomplete transfer of behavioural benefits from motor learning.

As a note of caution it should be stated that any transfer of effects of practicing the AAT tasks with the right dominant arm to the left non-dominant arm had not been tested in this experiment, and might differ from the observations that had been made here.

\subsection{Effects of cTBS on motor learning}

cTBS after prolonged training might interfere with any ongoing learning dynamics that occur within a ses- 
sion or might even more profoundly affect the level of skilfulness that had already been achieved after prolonged training. The former would shed light into the ongoing dynamics of learning after prolonged training. The latter might provide important information that could have implications for the clinical use of cTBS in patients after learning and (partial) recovery has already been achieved or when applied over the non-lesioned hemisphere with its preserved skilfulness.

In this study, cTBS was applied during the training sessions on days 13 to 16 over either M1, S1, PMC or SMA (order was counterbalanced across participants). Its effect on motor behaviour was analysed with intra-session effects which examined how cTBS changed performance from the 1 st to the 2 nd half of each training session. There was no sham-stimulation session (sham coil or stimulation over non-motor area). Instead, the four stimulation sites were compared against each other; thus, effects of cTBS over each stimulation site were compared to all other stimulation sites as control condition.

While during the first days of practice of the AAT tasks, cTBS over both S1, M1 and PMC had previously been shown to have task-specific detrimental effects (Platz et al., 2012), this was no longer the case after prolonged practice ( 3 rd week) of the same tasks in the current experiment (compare Fig. 3). In addition, there was no overall detrimental effect of cTBS on skilled performance with these tasks after prolonged training. The question might be raised whether this could be a mere power issue. This is, however, not likely. First, we can make a direct comparison with the previous related experiment on early motor learning where we clearly found significant cTBS effects with even a smaller sample size (Platz et al., 2012). Second, based on the effect size from this study, we calculated a post-hoc power analysis for repeated measures ANOVA assuming alpha $=5 \%$ and beta $=20 \%$. This showed that even testing twice as many subjects would not have led to significant findings.

The only differential cTBS effect that was observed was a possibly beneficial effect of inhibitory cTBS over M1 on within-session improvement with the labyrinth task. Why any inhibitory influence on M1 should enhance visuomotor performance (labyrinth task) is not readily clear, the effect had not been postulated in advance and could be a false positive effect. Theoretically, it might, how- ever, be entertained that inhibition of a system that controls selective movement and feed forward control (M1) could have triggered more and for the labyrinth task relevant feedback-based strategies using parietal-premotor networks involved in visuomotor performance and feedback-based learning (Grafton et al., 1998; 2002; Grol et al., 2006). Such reasoning would, however, ask for more direct evidence.

Overall, the data of both studies (current and Platz et al., 2012) lends support to the notion that during early stages of motor learning different sensorimotor cortical areas (including M1, S1 and PMC) are critically involved in sensorimotor learning, while their influence diminishes once motor learning has consolidated after a couple of weeks of extended practice. Indeed, the entire associative anterior premotor cortico-basal ganglia circuit is activated during the early stages of learning; once learned after extended practice motor skills are then, more likely to be stored in the sensorimotor cortico-basal ganglia circuit (Lehéricy et al., 2005); a differential set of cortical (e.g. M1) and subcortical structures in the basal ganglia and the cerebellum might then become more relevant and is thought to maintain a speedy representation of these motor skills when they are well learned and have become automatic (see also Jueptner et al., 1997; Seitz and Roland, 1992; Toni et al., 2001).

Further, there was no indication that cTBS as a type of inhibitory rTMS would significantly impair overlearnt skilful motor behaviour. This is a clinically relevant observation since inhibitory rTMS could play a role in stroke rehabilitation, especially when applied to the non-lesioned hemisphere (Platz and Rothwell, 2010). When inhibitory rTMS had been applied to the non-lesioned hemisphere of stroke patients, dysfunctions of the lesioned hemisphere (paresis, aphasia, neglect) could be improved. The current evidence might be reassuring indicating that cTBS of the nonlesioned hemisphere would not necessarily imply deterioration of skilfulness that is under control of this hemisphere.

\section{Conclusion}

This study on prolonged motor training indicated that the AAT induces substantial and robust motor learning in young healthy subjects by improving 
performance of different independent motor abilities, i.e. aiming, speed, steadiness, and visuomotor tracking that had been trained in parallel, with transfer to a non-trained motor tasks, and transfer to the non-trained arm. From the clinical perspective it is important to know that a 3 week course of AAT induces motor learning in terms of maintenance (retention) and generalisation (transfer) of training effects.

Detrimental effects of cTBS as previously shown when applied over S1, M1, and PMC within the first days of practice could no longer be substantiated when TMS was applied after prolonged motor practice.

\section{Acknowledgments}

This work was funded by the European Union (ImpactG Project, FP7-REGPOT-2008-1, Grant agreement no.: 229750, awarded to T.P.), the European Research Council under the European Union's Seventh Framework Programme (FP7/2007-2013) / ERC Grant agreement $\mathrm{n}^{\circ}$ (263472], awarded to A.T.S.), and by the German Research Association (DFG; LO 795/7, awarded to M.L. and T.P.). The sponsors had no role in study design; in the collection, analysis, and interpretation of data; in the writing of the report; or in the decision to submit the paper for publication.

\section{References}

Ashe, J., Lungu, O.V., Basford, A.T. \& Lu, X. (2006). Cortical control of motor sequences. Curr Opin Neurobiol, 16, 213-221.

Cohen, J. (1988). Statistical power analysis for behavioural sciences (2nd edition), Erlbaum, Hillsdale. NJ.

Grafton, S.T., Hazeltine, E. \& Ivry, R.B. (1998). Abstract and effector-specific representations of motor sequences identified with PET. J Neurosci, 18, 9420-9428.

Grafton, S., Hazeltine, E. \& Ivry, R. (2002). Motor sequence learning with the nondominant left hand. Exp Brain Res, 146, 369-378.

Grafton, S.T., Schmitt, P., Van Horn, J. \& Diedrichsen, J. (2008). Neural substrates of visuomotor learning based on improved feedback control and prediction. Neuroimage, 39, 1383-1395.

Grol, M.J., de Lange, F.P., Verstraten, F.A., Passingham, R.E. \& Toni, I. (2006). Cerebral changes during performance of overlearned arbitrary visuomotor associations. J Neurosci, 26, 117-125.

Isoda, M. \& Tanji, J. (2004). Participation of the primate presupplementary motor area in sequencing multiple saccades. $J$ Neurophysiol, 92, 653-659.
Jueptner, M., Frith, C.D., Brooks, D.J., Frackowiak, R.S. \& Passingham, R.E. (1997). Anatomy of motor learning. II. Subcortical structures and learning by trial and error. J Neurophysiol, 77, 1325-1337.

Keetch, K.M., Lee, T.D. \& Schmidt, R.A. (2008). Especial skills: Specificity embedded within generality. Journal of Sport and Exercise Psychology, 30, 723-736.

Lehéricy, S., Benali, H., Van de Moortele, P.F., Pélégrini-Issac, M., Waechter, T., Ugurbil, K. \& Doyon, J. (2005). Distinct basal ganglia territories are engaged in early and advanced motor sequence learning. Proc Natl Acad Sci USA, 102, 1256612571.

Lu, X. \& Ashe, J. (2005). Anticipatory activity in primary motor cortex codes memorized movement sequences. Neuron, 45, 967-973.

Maas, E., Robin, D.A., Austermann Hula, S.N., Freedman, S.E., Wulf, G., Ballard, K.J. \& Schmidt, R.A. (2008). Principles of motor learning in treatment of motor speech disorders. Am J Speech Lang Pathol, 17, 277-298.

Mathiowetz, V., Weber, K., Kashman, N. \& Volland, G. (1985). Adult norms for the Nine Hole Peg Test of finger dexterity. Occupational Therapy Journal of Research, 5, 24-38.

Matsuzaka, Y., Picard, N. \& Strick, P.L. (2007). Skill representation in the primary motor cortex after long-term practice. $J$ Neurophysiol, 97, 1819-1832.

Oldfield, R.C. (1971). The assessment and analysis of handedness: The Edinburgh inventory. Neuropsychologia, 9, 97-113.

Park, J.W., Kim, Y.H., Jang, S.H., Chang, W.H., Park, C.H. \& Kim, S.T. (2010). Dynamic changes in the cortico-subcortical network during early motor learning. NeuroRehabilitation, 26, 95-103.

Platz, T., Brown, R.G. \& Marsden, C.D. (1998). Training improves the speed of aimed movements in Parkinson's disease. Brain, $121,505-514$.

Platz, T. (2004). Impairment-oriented training (IOT) - scientific concept and evidence-based treatment strategies. Restor Neurol Neurosci, 22, 301-315.

Platz, T., van Kaick, S., Mehrholz, J., Leidner, O., Eickhof, C. \& Pohl, M. (2009). Best conventional therapy versus modular Impairment-oriented training (IOT) for arm paresis after stroke: A single blind, multi-centre randomized controlled trial. Neurorehabilitation and Neural Repair, 23, 706-716.

Platz, T. \& Rothwell, J.C. (2010). Brain stimulation and brain repair - rTMS: From animal experiment to clinical trials - what do we know? Restor Neurol Neurosci, 28, 387-398.

Platz, T., Roschka, S., Christel, M.I., Duecker, F., Rothwell, J.C. \& Sack, A. (2012). Motor skill learning and the specific relevance of the cortical motor system - a combined behavioural training and theta burst TMS study, Restor Neurol Neurosci, in press.

Schmidt, R.A. (1975). A schema theory of discrete motor skill learning. Psychological Review, 82, 225-260.

Schmidt, R.A. \& Lee, T.D. (2005). Motor control and learning: A behavioral emphasis (4th ed.), Human Kinetics, Champaign, IL.

Seidler, R.D. (2010). Neural correlates of motor learning, transfer of learning, and learning to learn. Exerc Sport Sci Rev, 38, 3-9. 
Seitz, R.J. \& Roland, P.E. (1992). Learning of sequential finger movements in man: A combined kinematic and Positron Emission Tomography (PET) Study. Eur J Neurosci, 4, 154165.

Shima, K. \& Tanji, J. (2000). Neuronal activity in the supplementary and presupplementary motor areas for temporal organization of multiple movements. J Neurophysiol, 84, 2148-2160.
Stockel, T. \& Weigelt, M. (2011). Brain lateralisation and motor learning: Selective effects of dominant and non-dominant hand practice on the early acquisition of throwing skills. Laterality, 14, 1-20.

Tanji, J. (2001). Sequential organization of multiple movements: Involvement of cortical motor areas. Anпи Rev Neurosci, 24, 631-651. 